\title{
A multi-centre, randomized, double-blind, placebo-controlled clinical trial of the efficacy and safety of chloroquine phosphate, hydroxychloroquine sulphate and lopinavir/ritonavir for the treatment of COVID-19 in Lagos State: study protocol for a randomized controlled trial
}
A. Abayomi ${ }^{1}$, A. Osibogun ${ }^{2}$, O. Ezechi ${ }^{3}$, K. Wright ${ }^{4}$, B. Ola ${ }^{4}$, O. Ojo ${ }^{5}$, Y. Kuyinu ${ }^{4}$, E. Zamba ${ }^{6}$, H. Abdur-Razzaq ${ }^{1}$,
O. A. Erinoso $0^{5}$ and S. E. Anya ${ }^{1 *}$ (D)

\begin{abstract}
Background: The coronavirus disease 2019 (COVID-19) is caused by the severe acute respiratory syndrome coronavirus 2 (SARS-CoV-2) that was first identified in Wuhan, Hubei, China, in December 2019. It was recognized as a pandemic by the World Health Organization on 11 March 2020. Outbreak forecasting and mathematical modelling suggest that these numbers will continue to rise. Early identification of effective remedies that can shorten the duration and severity of illness is critical for Lagos State, which is the epi-centre of the disease in Nigeria.

Methods: This is a multi-centre, randomized, double-blind placebo-controlled superiority trial. The study investigates the efficacy of chloroquine phosphate, hydroxychloroquine sulphate and lopinavir/ritonavir added on to standard of care compared to standard of care only in patients with COVID-19 disease. The primary outcome is the clinical status of patients measured using a 7-point ordinal scale at day 15. Research participants and clinicians will be blinded to the allocated intervention. Outcome measures will be directly assessed by clinicians. Statistical analysis will be done by a team blinded to the identity and allocation of research participants. Data analysis will follow intention-to-treat methods, using R software.

Discussion: The current study is of strategic importance for Lagos State in potentially curbing the health, social and economic burden of COVID-19 disease. Should the current study demonstrate that either of the three intervention drugs is more efficacious than standard therapy alone, the State Ministry of Health will develop an evidence-based guideline for the management of COVID-19 in Lagos State. The findings will also be shared nationally and with other states which may lead to a standardized national guideline for the treatment of COVID-19 in Nigeria.
\end{abstract}

\footnotetext{
* Correspondence: sea.anya@gmail.com

${ }^{1}$ Lagos State Ministry of Health, Alausa, Ikeja, Lagos State, Nigeria

Full list of author information is available at the end of the article
}

(c) The Author(s). 2021 Open Access This article is licensed under a Creative Commons Attribution 4.0 International License, which permits use, sharing, adaptation, distribution and reproduction in any medium or format, as long as you give appropriate credit to the original author(s) and the source, provide a link to the Creative Commons licence, and indicate if changes were made. The images or other third party material in this article are included in the article's Creative Commons licence, unless indicated otherwise in a credit line to the material. If material is not included in the article's Creative Commons licence and your intended use is not permitted by statutory regulation or exceeds the permitted use, you will need to obtain permission directly from the copyright holder. To view a copy of this licence, visit http://creativecommons.org/licenses/by/4.0/. The Creative Commons Public Domain Dedication waiver (http://creativecommons.org/publicdomain/zero/1.0/) applies to the data made available in this article, unless otherwise stated in a credit line to the data. 
Trial registration: Pan African Clinical Trials Register PACTR202004801273802. Registered prospectively on April 2, 2020 Keywords: COVID-19, SARS-CoV-2, 2019-nCoV, Chloroquine, Hydroxychloroquine, Lopinavir/ritonavir, Lagos, Nigeria

\section{Administrative information}

Note: the numbers in curly brackets in this protocol refer to SPIRIT checklist item numbers. The order of the items has been modified to group similar items (see http://www.equator-network.org/reporting-guidelines/ spirit-2013-statement-defining-standard-protocol-itemsfor-clinical-trials/) (Table 1).

\section{Introduction}

\section{Background and rationale $\{6 a\}$ Study rationale}

The severe acute respiratory syndrome coronavirus 2 (SARS-CoV-2) causes the coronavirus disease 2019 (COVID-19), and it was first detected in Wuhan, China, in December 2019 [1]. COVID-19 disease was recognized as a pandemic by the World Health Organization (WHO) less than 3 months after on the 11th of March 2020 [2]. By July 1, 2020, more than 10,357,662 cases of COVID-19 had been reported, with about 508,055 deaths globally [3, 4]. COVID-19 outbreak forecasts and mathematical models suggest that the size of the population affected will continue to rise [5] in the coming months.

The virus is typically spread from one person to another via respiratory droplets produced during speech, coughing and sneezing [6]. Primarily, it spreads when people are in close contact, but it may also spread when one touches a contaminated surface and then their eyes, nose or mouth. It is most contagious when people are symptomatic, although spread may be possible before symptoms appear. The time between exposure and symptom onset is typically around 5 days but may range from 2 to 14 days. Fever and chills and dry cough, as well as shortness of breath, are commonly reported symptoms [1]. Complications may include pneumonia and acute respiratory distress syndrome. A WHO investigative report indicates that age over 60 years, diabetes mellitus, cardiovascular disease, chronic respiratory disease and cancer are associated with the highest risk of severe disease and death [1].

In Nigeria, a total of 26,484 cases and 603 deaths had been reported as of 1 July 2020 with Lagos alone accounting for about half of the cases [7]. The high proportion of cases contributed by Lagos State is likely due to the fact that it is the commercial capital with a large number of international travellers and has COVID-19 testing facilities and a very large population compared to other states.

Coronavirus is a type of single-stranded positivestrand RNA virus with an envelope [8]. Currently, there is no specific therapy for coronavirus infections. Only a limited number of treatment studies have been conducted because most human coronavirus strains cause self-limiting disease and care is supportive. However, after the severe acute respiratory syndrome (SARS) coronavirus was identified in 2002 and caused a large global outbreak, there was an increased interest in the development of specific therapeutic agents. SARS-CoV case-

Table 1 Administrative information for Lagos COVID-19 Treatment Trial (LACTT)

\begin{tabular}{|c|c|}
\hline Title $\{1\}$ & $\begin{array}{l}\text { A Multi-Center, Randomized, Double-Blind, Placebo-Controlled Clinical Trial of the Efficacy and Safety of } \\
\text { Chloroquine Phosphate, Hydroxychloroquine Sulphate and Lopinavir/Ritonavir for the Treatment of COVID- } \\
19 \text { in Lagos State, Nigeria }\end{array}$ \\
\hline Trial registration $\{2 \mathrm{a}$ and $2 \mathrm{~b}\}$. & $\begin{array}{l}\text { The trial was registered with the Pan African Clinical Trials Register on April 2nd, } 2020 \text { with registration } \\
\text { number PACTR202004801273802. The URL of the registry record is https://pactr.samrc.ac.za/TrialDisplay. } \\
\text { aspx?TriallD=10928. }\end{array}$ \\
\hline Protocol version $\{3\}$ & $20^{\text {th }}$ July 2020 , version number: 3.0 . \\
\hline Author details $\{5 \mathrm{a}\}$ & $\begin{array}{l}{ }^{1} \text { Lagos State Ministry of Health, Lagos, Nigeria } \\
{ }^{2} \text { Lagos State Primary Health Care Board, Lagos, Nigeria } \\
{ }^{3} \text { Nigerian Institute of Medical Research, Lagos, Nigeria } \\
{ }^{4} \text { Lagos State University College of Medicine, Lagos, Nigeria } \\
{ }^{5} \text { Lagos State University Teaching Hospital, Lagos, Nigeria } \\
{ }^{6} \text { Lagos State Health Management Agency, Lagos, Nigeria }\end{array}$ \\
\hline $\begin{array}{l}\text { Name and contact information for the } \\
\text { trial sponsor }\{5 b\}\end{array}$ & $\begin{array}{l}\text { Dr. Olusegun Ogboye, BSc, MBBS, MPH } \\
\text { Permanent Secretary, Lagos State Ministry of Health, Lagos, Nigeria. } \\
\text { email: olusegun.ogboye@lagosstate.gov.ng }\end{array}$ \\
\hline Role of sponsor $\{5 c\}$ & $\begin{array}{l}\text { The study sponsor and funder do not have a role in the design; collection, management, analysis, and } \\
\text { interpretation of data; writing of the report; and the decision to submit the this or other manuscripts for } \\
\text { publication. }\end{array}$ \\
\hline
\end{tabular}


patients were treated with corticosteroids, chloroquine phosphate, hydroxychloroquine, convalescent plasma, and lopinavir or ritonavir, and, except for ribavirin, many of these agents have in vitro preclinical data that support their efficacy [9-19]. Since the SARS outbreak, new therapeutic agents targeting viral entry proteins, proteases, polymerases and methyltransferases have been tested; however, none of them has been shown to be efficacious in clinical trials [20-22].

There are reports about the potential value of a number of drugs [9-12, 23]. Of the various therapeutic interventions that have been suggested as being potentially effective, chloroquine stands out as an attractive option for the treatment of COVID-19 in Nigeria, if found to be efficacious. It has been used extensively for the treatment of malaria in Nigeria and is low-cost. Clinically, it has been reported that chloroquine improves outcomes in patients with COVID-19 [24-26]. However, robust clinical trials are still required to prove their effectiveness and safety in the treatment of COVID-19 due to limitations in the design of reported clinical studies. Furthermore, there are recent reports of chloroquine poisoning following announcements of its potential effectiveness in the treatment of COVID-19 [27]. This compelled a notice by the Nigeria Centre for Disease Control (NCDC) publicly stating that chloroquine is not an approved treatment for COVID-19 [28].

Chloroquine has been used clinically since 1944, primarily for the prevention and treatment of malaria [29]. More recently, it has been shown to have antiviral activity against HIV-1, avian influenza H5N1, HCoV-229E, hepatitis B and herpes simplex type 1 viruses [29-32]. Chloroquine's anti-SARS-CoV activity involves a variety of mechanisms that inhibit virus replication such as suppressing phosphatidylinositol binding clathrin assembly protein (PICALM), inhibiting endosomal acidification required for virus/cell fusion and reducing terminal glycosylation of angiotensin-converting enzyme 2 (ACE2) receptors [31, 33, 34]. Chloroquine also suppresses tumour necrosis factor alpha and interleukin 6, which mediate the inflammatory complications of viral infection [29].

The inhibition of infection at the cellular level has been shown to occur at a $50 \%$ inhibitory concentration (IC50) of $8.8 \pm 1.2 \mu \mathrm{M}$ for SARS-CoV [31] and 50\% maximal effective concentration $(E C 50)$ of $1.13 \mu \mathrm{M}$ for SARS-CoV-2 [9]. Furthermore, an EC90 of $6.9 \mu \mathrm{M}$ was achieved for SAR-CoV-2 [9]. These findings are significant because they suggest that therapeutic doses needed for effective anti-SARS-CoV-2 activity are similar to doses used in the treatment of malaria, which is endemic in Nigeria, and rheumatoid arthritis [31, 35]. Hydroxychloroquine has not been studied to the same extent, but it appears to have more potent in vitro efficacy [36].
These concentrations of chloroquine are significantly lower than the $50 \%$ cytotoxic concentration (CC50) which ranged from greater than $100 \mu \mathrm{m}$ to $261.3 \pm 14.5 \mu \mathrm{m}$ with selectivity index from 30 to more than $88[9,31]$.

Despite the promising results from in vitro studies, chloroquine has not been shown conclusively to be efficacious in the treatment of COVID-19. However, it is now part of the recommendations for treatment in several countries while awaiting results of planned and ongoing clinical trials $[23,36]$. These recommendations have been informed by two main sources-an expert consensus on chloroquine phosphate for treatment of COVID-19 from China and a clinical study undertaken in France [26, 37]. These and one other small study reported from China suggest that chloroquine and hydroxychloroquine prevent the worsening of pneumonia, facilitate the clearance of the virus and shorten the duration of COVID-19 [24-26, 37].

There are very few clinical studies on the efficacy of chloroquine phosphate or hydroxychloroquine sulphate. In the main study that has generated expectations of a cure, hydroxychloroquine appeared to lead to a rapid decline in nasopharyngeal viral load with negative viral cultures in almost all patients at day 5 [26]. The duration of hospital admission was also short averaging 5 days. Although impressive, this evidence is considered weak [23], due to the methodological design of the study which had a small sample size and no control group.

Similar to chloroquine phosphate and hydroxychloroquine sulphate, there are few clinical studies investigating the effect of lopinavir/ritonavir in the treatment of COVID-19 disease [38]. The combination of antiviral serum protease inhibitors lopinavir and ritonavir has been touted as a potential therapy for COVID-19 disease. Both drugs have historically been used to manage human immunodeficiency disease type 1 (HIV-1) and found to have an in vitro inhibitory effect on the SARS$\mathrm{CoV}$ virus $[11,23,39,40]$. The antivirals work synchronously with ritonavir which inhibits the enzyme cytochrome P450 and by effect increases the plasma half-life of lopinavir [11].

Cao et al. [38] conducted an open-label randomized controlled trial in hospitalized patients using lopinavir/ ritonavir in combination with standard care therapy. The study found that lopinavir/ritonavir in combination with standard therapy did not provide a clinical benefit or reduce mortality compared to standard therapy alone. However, the study was not blinded, meaning the knowledge of the assigned treatment could have biased the findings of the outcome assessors.

The relatively high morbidity associated with COVID19 and potentially inappropriate use of publicized remedies means that identifying effective medications that can reduce the duration and severity of illness is a high 
priority globally. The World Health Organization is taking steps towards a multi-country clinical trial of potential therapeutic agents [41]. This study is being undertaken as part of global efforts to identify effective specific therapeutic agents for COVID-19.

Broadly, this study, is asking three different questions, namely (i) does chloroquine phosphate safely improve patient outcome when added to the standard treatment for COVID-19 compared to the addition of placebo?; (ii) does hydroxychloroquine sulphate safely improve patient outcome when added to the standard treatment for COVID-19 compared to the addition of placebo?; and (iii) does lopinavir/ritonavir safely improve patient outcome when added to the standard treatment for COVID-19 compared to the addition of placebo?

\section{Importance of the Lagos COVID-19 Treatment Trial}

Early identification of effective remedies that can shorten the duration and severity of illness is critical for Lagos State and Nigeria. The COVID-19 pandemic places an enormous burden on the Nigerian health system which already faces significant resource constraints. There is a limited number of hospital beds, especially, for infectious diseases requiring isolation and severely ill patients who need high dependency or intensive care. The availability of such treatment would reduce morbidity and mortality and increase the availability of hospital beds, thereby, enabling management of the epidemic without compromising care for patients with other diseases. Effective treatment may also lead to an earlier resolution of the pandemic.

Testing these drugs in a robust trial is important because it would help determine conclusively whether or not they are efficacious. If they are, then the scenario above applies, while if they are not then efforts can be redirected to examine other potential remedies and resources would not be spent on ineffective therapies.

\section{Objectives $\{7\}$}

1. To evaluate the clinical efficacy of chloroquine phosphate, hydroxychloroquine sulphate and lopinavir/ritonavir added on to standard therapy relative to standard therapy alone for treatment of individuals with laboratory-confirmed COVID-19

1.1. To evaluate the clinical efficacy of chloroquine phosphate added on to standard therapy relative to standard therapy alone for treatment of individuals with laboratory-confirmed COVID-19

1.2. To evaluate the clinical efficacy of hydroxychloroquine sulphate added on to standard therapy relative to standard therapy alone for treatment of individuals with

laboratory-confirmed COVID-19

1.3. To evaluate the clinical efficacy of lopinavir/ ritonavir added on to standard therapy relative to standard therapy alone for treatment of individuals with laboratory-confirmed COVID-19

The secondary objectives are:

1. To evaluate the effect of chloroquine phosphate, hydroxychloroquine sulphate and lopinavir/ ritonavir added on to standard therapy compared to standard therapy on the rate of progression/ resolution of COVID-19

1.1. To evaluate the effect of chloroquine phosphate added on to standard therapy compared to standard therapy on the rate of progression/ resolution of COVID-19

1.2. To evaluate the effect of hydroxychloroquine sulphate added on to standard therapy compared to standard therapy on the rate of progression/resolution of COVID-19

1.3. To evaluate the effect of lopinavir/ritonavir added on to standard therapy compared to standard therapy on the rate of progression/ resolution of COVID-19

2. 2. To evaluate the safety of chloroquine phosphate, hydroxychloroquine sulphate and lopinavir/ ritonavir added on to standard therapy compared to standard therapy in the treatment of individuals with laboratory-confirmed COVID-19

2.1. To evaluate the safety of chloroquine phosphate added on to standard therapy compared to standard therapy in the treatment of individuals with laboratory-confirmed COVID-19

2.2. To evaluate the safety of hydroxychloroquine sulphate added on to standard therapy compared to standard therapy in the treatment of individuals with laboratory-confirmed COVID-19

2.3. To evaluate the safety of lopinavir/ritonavir added on to standard therapy compared to standard therapy in the treatment of individuals with laboratory-confirmed COVID-19

\section{Trial design $\{\mathbf{8}\}$}

This is a multi-centre, randomized, double-blind, placebo-controlled multi-arm clinical trial with a parallel group and superiority design. There are three treatment arms and one control arm with equal distribution of participants between the arms. If the intervention drug for any arm is not available, then participants will be allocated equally to each of the available arms. 


\section{Methods: participants, interventions and outcomes}

Study setting $\{9\}$

This trial will take place at health facilities designated for COVID-19 patients in Lagos State, Nigeria. The LACTT trial will be conducted at three of the health facilities designated for COVID-19 patients in Lagos State, Nigeria. The centres are Lagos Mainland Infectious Disease Hospital, Yaba; the Eti-Osa COVID-19 Isolation and Treatment Centre; and the Onikan COVID-19 Isolation and Treatment Centre Lagos State. The three centres manage patients domiciled in Lagos State, based on a similar state-wide protocol which aligns with the Nigeria Centre for Disease Control guideline for testing and management of COVID-19 disease. The proposed sample size distribution across the three centres is 200 participants per centre.

\section{Eligibility criteria $\{10\}$ Inclusion criteria}

In order to be eligible to participate in this study, a patient must meet all of the following criteria: (1) provide written informed consent prior to initiation of any study procedures; (2) understand and agree to comply with planned study procedures; (3) agree to the collection of oropharyngeal swabs and venous blood per protocol; (4) be male or non-pregnant female adult $\geq 18$ years of age at the time of enrolment; and (5) have laboratoryconfirmed SARS-CoV-2 infection as determined by RTPCR in any specimen no more than $72 \mathrm{~h}$ prior to randomization. Both asymptomatic and symptomatic cases (mild/moderate/severe) are eligible for enrolment.

\section{Exclusion criteria}

An individual who meets any of the following criteria will be excluded from participation in this study:

1. ALT/AST $>5$ times the upper limit of normal

2. Stage 4 severe chronic kidney disease or requiring dialysis (i.e. eGFR < 30)

3. Pregnant or breast feeding

4. Anticipated transfer to another facility which is not a study site within $72 \mathrm{~h}$

5. Participants with known haematological diseases, e.g. G6PD deficiency

6. Participants with chronic liver and kidney disease and reaching end-stage

7. Participants with arrhythmia and chronic heart disease

8. Participants known to have retinal disease or hearing loss

9. Participants known to have a mental illness

10. Skin disorders (including rash, dermatitis, psoriasis)
11. Allergy to any study medication (4-aminoquinoline or lopinavir/ritonavir)

\section{Lifestyle consideration}

Participants will be advised to refrain from alcohol and tobacco use during the period of study.

\section{Who will take informed consent? \{26a\}}

Informed consent will be taken by trained clinical research assistants (CRAs). Discussions of essential information about the research will include the study's purpose, duration, experimental procedures, alternatives, risks and benefits, and subjects will have the opportunity to ask questions and have them answered. There will be interpreters at the study sites for potential participants who cannot communicate in English.

Additional consent provisions for collection and use of participant data and biological specimens $\{26 \mathrm{~b}\}$

Potential participants will be asked for consent to collect additional blood by CRAs, the use of residual specimens and samples for secondary research. Extra blood will be drawn for secondary research by CRAs during designated days when study blood samples are obtained.

The stored samples will be labelled with codes to maintain confidentiality. Research with identifiable samples and data may occur as needed; however, subject confidentiality will be maintained as described for this protocol and with HREC approval. Samples designated for secondary research use may be used for understanding the SARS-CoV-2 infection, the immune response to this infection, and the effect of therapeutics on these factors. Samples will not be sold. Although the results of any future research may be patentable or have commercial profit, participants will have no legal or financial interest in any commercial development resulting from any future research.

There are no direct benefits to the participant for extra specimens collected or from the secondary research. No results from secondary research will be entered into the participant's medical record. Incidental findings will not be shared with the participant, including medically actionable incidental findings, unless required by law. Participants may withdraw permission to use samples for secondary use at any time. They will need to contact the study site and the samples will be removed from the study repository after this study is completed and documentation will be completed that outlines the reason for withdrawal of permission for secondary use of samples.

\section{Explanation for the choice of comparators $\{6 b\}$}

The comparator for this study is the standard of care at each of the study sites. 
The standard supportive for adults with COVID-19 are as stated below (Table 2).

\section{Intervention description $\{11$ a}

The dose and route of administration of the trial drugs are fixed. The medications will be taken with food. Missed doses for the day will not be made up. All intervention medications will be administered with the standard of care.

\section{Arm 1}

In the chloroquine phosphate arm of the study, participants will receive chloroquine phosphate two $250 \mathrm{mg}$ tablets orally twice a day $(12 \mathrm{~h} / 24 \mathrm{~h})$ for 7 days, two hydroxychloroquine placebo tablets orally twice a day (12h/24h) for 7 days and two lopinavir/ritonavir placebo tablets orally twice a day $(12 \mathrm{~h} / 24 \mathrm{~h})$ for 14 days.

\section{Arm 2}

In the hydroxychloroquine arm, participants will receive hydroxychloroquine sulphate two $200 \mathrm{mg}$ tablets orally twice a day $(12 \mathrm{~h} / 24 \mathrm{~h})$ for 7 days, two chloroquine placebo tablets orally twice a day $(12 \mathrm{~h} / 24 \mathrm{~h})$ for 7 days and two lopinavir/ritonavir placebo tablets orally twice a day (12h/24h) for 14 days.

\section{Arm 3}

In the lopinavir/ritonavir arm, participants will receive two tablets of lopinavir $200 \mathrm{mg} /$ ritonavir $50 \mathrm{mg}$ combination tablet orally twice a day $(12 \mathrm{~h} / 24 \mathrm{~h})$ for 14 days, two chloroquine placebo tablets orally twice a day $(12 \mathrm{~h} / 24 \mathrm{~h})$ for 7 days and two hydroxychloroquine placebo tablets orally twice a day $(12 h / 24 h)$ for 7 days.

\section{Arm 4}

In the control arm of the study, participants will receive two chloroquine placebo tablets orally twice a day $(12 \mathrm{~h} /$ 24h) for 7 days, two hydroxychloroquine placebo tablets orally twice a day $(12 \mathrm{~h} / 24 \mathrm{~h})$ for 7 days and two lopinavir/ritonavir placebo tablets orally twice a day $(12 \mathrm{~h} / 24 \mathrm{~h})$ for 14 days.

If any of the treatment arms is not available, the placebo for the relevant drug will not be administered to participants in the other arms.

\section{Criteria for discontinuing or modifying allocated interventions $\{11 \mathrm{~b}\}$}

If the estimated creatinine clearance decreases by more than $\geq 50 \%$ from baseline, the study interventions (oral chloroquine phosphate, hydroxychloroquine sulphate and lopinavir/ritonavir) will not be given. The interventions may be resumed when the estimated creatinine clearance returns to baseline. If the liver function tests (ALT and/or AST) increase to $>2$ times upper limits of normal, the dose of chloroquine phosphate, hydroxychloroquine sulphate and lopinavir/ritonavir will not be administered. Dosing may be resumed when the ALT and/or AST returns to baseline. Dosing may be given

Table 2 Standard of care for COVID-19 at study sites

\begin{tabular}{|c|c|}
\hline Standard supportive therapy & Explanation \\
\hline $\begin{array}{l}\text { Supplemental oxygen therapy immediately to patients with severe acute } \\
\text { respiratory infection (SARI) and respiratory distress, hypoxaemia or shock. }\end{array}$ & $\begin{array}{l}\text { - Commence oxygen therapy at } 5 \mathrm{~L} / \mathrm{min} \text { and titrate flow rates to reach } \\
\text { target } \mathrm{SpO}_{2} \geq 90 \% \text { in non-pregnant adults and } \mathrm{SpO}_{2} \geq 92-95 \% \text { in preg- } \\
\text { nant patients. } \\
\text { - Children with emergency signs, such as breathing difficulty, obstruction } \\
\text { or apnea, severe respiratory distress, central cyanosis, shock, coma or } \\
\text { convulsions, should receive oxygen therapy during resuscitation to } \\
\text { target } \mathrm{SpO}_{2} \geq 94 \% \text {; otherwise, the target } \mathrm{SpO}_{2} \text { is } \geq 90 \% \text {. } \\
\text { - Wards and outpatients where patients with } \mathrm{SARI} \text { are cared for are } \\
\text { equipped with pulse oximeters, functioning oxygen systems and } \\
\text { disposable, single-use, oxygen-delivering interfaces (nasal cannula, simple } \\
\text { face mask and mask with reservoir bag). } \\
\text { - Contact precautions will be taken when handling contaminated oxygen } \\
\text { interfaces of patients with SARS-COV-2 infection. }\end{array}$ \\
\hline $\begin{array}{l}\text { Conservative fluid management in patients with SARI when there is no } \\
\text { evidence of shock. }\end{array}$ & $\begin{array}{l}\text { - Patients with SARI will be treated cautiously with intravenous fluids, } \\
\text { because aggressive fluid resuscitation may worsen oxygenation. }\end{array}$ \\
\hline $\begin{array}{l}\text { Empiric antimicrobials to treat all likely pathogens causing SARI. } \\
\text { Antimicrobials (Augmentin } 650 \mathrm{mg} \text { tablets } 12 \mathrm{hhrly} \text { for } 24 \mathrm{~h} \text { or Augmentin } \\
\text { IV } 1 \mathrm{~g} \mathrm{q} 24 \mathrm{~h} \text { ) within } 1 \mathrm{~h} \text { of initial patient assessment for patients with } \\
\text { evidence of URTI or sepsis. }\end{array}$ & $\begin{array}{l}\text { - The clinicians will administer appropriate empiric antimicrobials within } 1 \\
\text { h of identification of sepsis or URTI. } \\
\text { - Empiric antibiotic treatment will be based on the clinical diagnosis } \\
\text { (community-acquired pneumonia, healthcare-associated pneumonia [if } \\
\text { infection was acquired in healthcare setting] or sepsis). Empiric therapy } \\
\text { will be de-escalated on the basis of microbiology results and clinical } \\
\text { judgment. }\end{array}$ \\
\hline $\begin{array}{l}\text { - Zinc sulphate tablets } 100 \mathrm{mg} \text { daily } \\
\text { - Calcium tablets } 300 \mathrm{mg} \text { daily } \\
\text { - Vitamin C tablets } 1 \mathrm{~g} \text { daily } \\
\text { - Vitamin D tablets } 50 \mathrm{mcg} \text { daily }\end{array}$ & - Given to all individuals with COVID-19 \\
\hline
\end{tabular}


later the same day. If a day's dosing is missed, it will not be made up.

If any of the following occur, the dose of chloroquine phosphate or hydroxychloroquine sulphate or lopinavir/ ritonavir will be stopped and will not be restarted:

- ALT $\geq 3 \times$ upper limits of normal and bilirubin $\geq 2 \times$ upper limits of normal

- ALT and/or AST increases to > 5 times upper limits of normal

\section{Strategies to improve adherence to interventions $\{11 \mathrm{c}\}$}

Participants will be directly observed taking their medications by clinical nurses and CRAs at the study sites to encourage and monitor adherence.

\section{Relevant concomitant care permitted or prohibited during the trial $\{11 \mathrm{~d}\}$}

Non-supportive therapy prior to enrolment is permitted. Concomitant therapy for co-morbidities such as hypertension, diabetes, rheumatoid arthritis etc. will be permitted and documented. In addition, the list of participants' medications will be assessed (from medical history) from at least 30 days prior to enrolment to the 15 th day after enrolment.

\section{Provisions for post-trial care $\{30\}$}

Study personnel will try to reduce, control and treat any harm from trial participation. Immediate medical treatment will be provided at Lagos Mainland Hospital Yaba. As needed, referrals to appropriate healthcare facilities will be provided to the subject. The site study physician will then determine if an injury occurred as a direct result of the tests or treatments that are done for this trial.

If it is determined by the site study physician that an injury occurred to a subject as a direct result of the tests or treatments that are done for this trial, then referrals to appropriate healthcare facilities will be provided to the subject. In case of SAEs or other research-related injuries, the full cost of treatment and ensuing cost associated with any associated complications will be borne by study sponsors.

\section{Outcomes $\{12\}$}

COVID-19 is a new disease and our understanding of it is evolving. To align this study with other international studies, the objectives, endpoints and some other aspects of the WHO's Master Protocol [42] have been adopted as appropriate with modifications to suite the Lagos context. There are three objectives-one primary and two secondary objectives. Each objective has three subobjectives-one for comparing chloroquine phosphate added on to standard therapy and standard therapy alone; one for comparing hydroxychloroquine sulphate added on to standard therapy and standard therapy alone; and one for comparing lopinavir/ritonavir added on to standard therapy and standard therapy alone.

A special WHO committee developed the ordinal scale that will be used in this study for the primary endpoint [43]. This study uses a combined endpoint (based on the WHO 7-point ordinal scale) of both "clinical status and mortality" at day 15 . Hence, superiority can be demonstrated when either arm shows a significant effect on the variables for clinical status OR mortality by day 15 . This endpoint is particularly useful in our population given the low mortality rate, and a combined endpoint would increase the study power. The secondary endpoints will enable evaluation of the effect of the trial medications on the rate of change in the clinical condition of COVID-19 patients, as well as the safety of the trial medications (Table 3).

\section{Efficacy assessments}

The schedule of assessments (SOA) in Table 4 outlines the baseline and follow-up assessments that will be done.

\section{Measures of clinical status}

On each study day while hospitalized or in isolation, the following measure of clinical status will be assessed:

- Symptoms

- Vital signs

- Oxygen requirement

- Non-invasive mechanical ventilation (via mask)

- Mechanical ventilator requirement (via an endotracheal tube or tracheostomy tube)

\section{Ordinal scale}

The ordinal scale is an assessment of the clinical status at the first assessment of a given study day. Each day, the worse score for the previous day will be recorded, i.e. on day 3, the day 2 score is obtained and recorded as day 2 . The scale is as follows:

1. Not hospitalized, no limitations on activities

2. Not hospitalized, limitation on activities

3. Hospitalized, not requiring supplemental oxygen

4. Hospitalized, requiring supplemental oxygen

5. Hospitalized, on non-invasive ventilation or high flow oxygen devices

6. Hospitalized, on invasive mechanical ventilation

7. Death

\section{NEW score}

The NEW score is based on 7 clinical parameters (see Table 5) and is being used as an efficacy measure. This 
Table 3 LACTT objectives and endpoints

\section{Primary objectives \\ 1. To evaluate the clinical efficacy of chloroquine phosphate, hydroxychloroquine sulphate and lopinavir/ritonavir added on to standard therapy relative to standard therapy alone for treatment of individuals with laboratory-confirmed COVID-19 \\ 1.1 To evaluate the clinical efficacy of chloroquine phosphate added on to standard therapy relative to standard therapy alone for treatment of individuals with laboratory-confirmed COVID-19 \\ 1.2 To evaluate the clinical efficacy of hydroxychloroquine sulphate added on to standard therapy relative to standard therapy alone for treatment of individuals with laboratory-confirmed COVID-19 \\ 1.3 To evaluate the clinical efficacy of lopinavir/ritonavir added on to standard therapy relative to standard therapy alone for treatment of individuals with laboratory-confirmed COVID-19}

\section{Secondary objectives}

1. To evaluate the effect of chloroquine phosphate, hydroxychloroquine sulphate and lopinavir/ritonavir added on to standard therapy compared to standard therapy on the rate of progression/resolution of COVID-19

1.1 To evaluate the effect of chloroquine phosphate added on to standard therapy compared to standard therapy on the rate of progression/resolution of COVID-19

1.2 To evaluate the effect of hydroxychloroquine sulphate added on to standard therapy compared to standard therapy on the rate of progression/resolution of COVID-19

1.3 To evaluate the effect of lopinavir/ritonavir added on to standard therapy compared to standard therapy on the rate of progression/ resolution of COVID-19

2. To evaluate the safety of chloroquine phosphate, hydroxychloroquine sulphate and lopinavir/ritonavir added on to standard therapy compared to standard therapy in the treatment of individuals with laboratory-confirmed COVID-19

2.1 To evaluate the safety of chloroquine phosphate added on to standard therapy compared to standard therapy in the treatment of individuals with laboratory-confirmed COVID-19

2.2 To evaluate the safety of hydroxychloroquine sulphate added on to standard therapy compared to standard therapy in the treatment of individuals with laboratory-confirmed COVID-19

2.3 To evaluate the safety of lopinavir/ritonavir added on to standard therapy compared to standard therapy in the treatment of individuals with laboratory-confirmed COVID-19

\author{
Primary endpoints \\ Seven-point ordinal scale assessed on day 15 \\ 1. Not hospitalized, no limitations on activities \\ 2. Not hospitalized, limitation on activities \\ 3. Hospitalized, not requiring supplemental oxygen \\ 4. Hospitalized, requiring supplemental oxygen \\ 5. Hospitalized, on non-invasive ventilation or high flow oxygen devices \\ 6. Hospitalized, on invasive mechanical ventilation \\ 7. Death
}

\section{Secondary endpoints}

1. Clinical status on the 7-point ordinal scale on days 3, 5, 8, 11 and 29

2. National Early Warning Score assessed daily while hospitalized and on day 15

3. Duration of supplemental oxygen

4. Duration of mechanical ventilation

5. Duration of hospitalization or isolation

6. SARS-CoV-2 clearance time based on samples taken on days 3, 5, 8, 11 ,

15 and 29

1. Cumulative incidence of severe adverse events up to and including day 29

2. Changes in laboratory safety indices assessed on days 1, 8 and 15haemoglobin concentration, platelet count, white blood cell count, glucose, creatinine, total bilirubin, ALT and AST will be evaluated at the first assessment of a given study day. These parameters can be obtained from the hospital chart using the last measurement prior to the time of assessment. This is recorded for the day obtained, i.e. on day 4 , the day 3 score is obtained and recorded as day 3 .

\section{Further assessments}

Oropharyngeal swabs and blood/serum samples will be collected on days 1, 8 and 15 (while hospitalized or in isolation) and stored. Tests for viral shedding and antiviral antibodies are being developed and will be used to test the stored sample when they become available.

\section{Safety assessments}

Study procedures are specified in the SOA. A study physician will be responsible for all trial-related medical decisions.

- Physical examination
- A symptom-directed (targeted) physical examination will be performed to evaluate for any possible adverse event.

- Clinical laboratory evaluations

- Fasting is not required before collection of laboratory samples.

- Blood will be collected at the time points indicated in the SOA. Clinical laboratory parameters include WBC, $\mathrm{Hb}, \mathrm{PLT}, \mathrm{Cr}$, glucose, total bilirubin, AST and ALT.

If a physiologic parameter, e.g. vital signs, or laboratory value is outside of the protocol-specified range (Table 6), then the measurement may be repeated once if, in the judgment of the investigator, the abnormality is the result of an acute, short-term, rapidly reversible condition or was an error. A physiologic parameter may also be repeated if there is a technical problem with the measurement caused by malfunctioning, 
Table 4 Schedule of assessments

\begin{tabular}{|c|c|c|c|c|c|c|c|c|c|c|c|c|c|c|}
\hline \multirow{2}{*}{$\begin{array}{l}\text { Study period } \\
\text { Day } \pm \text { window }\end{array}$} & \multirow{2}{*}{$\begin{array}{l}\text { Screening } \\
-1 \text { or } 1\end{array}$} & \multirow{2}{*}{$\begin{array}{l}\text { Baseline } \\
1\end{array}$} & \multicolumn{12}{|c|}{ Follow-up days } \\
\hline & & & 2 & 3 & 4 & 5 & 6 & 7 & 8 & 9 & 10 & $11-14$ & $15 \pm 2$ & $29 \pm 3$ \\
\hline \multicolumn{15}{|l|}{ 1. Eligibility } \\
\hline Informed consent & $x$ & & & & & & & & & & & & & \\
\hline Demographics and medical history & $x$ & & & & & & & & & & & & & \\
\hline Lung auscultation & $x$ & & & & & & & & & & & & & \\
\hline Chest radiography & $x$ & & & & & & & & & & & & & \\
\hline Confirm SARS-CoV-2 results ${ }^{1}$ & $x$ & & & & & & & & & & & & & \\
\hline \multicolumn{15}{|l|}{ 2. Study interventions } \\
\hline Randomization & & $x$ & & & & & & & & & & & & \\
\hline Administration of study drugs ${ }^{2}$ & & $x$ & $x$ & $x$ & $x$ & $x$ & $x$ & $x$ & $x$ & $x$ & $x$ & $x$ & & \\
\hline \multicolumn{15}{|l|}{ 3. Study procedures } \\
\hline Vital signs & & $x$ & $x$ & $x$ & $x$ & $x$ & $x$ & $x$ & $x$ & $x$ & $x$ & $x$ & $x$ & \\
\hline Clinical assessment $^{3}$ & & $x$ & $x$ & $x$ & $x$ & $x$ & $x$ & $x$ & $x$ & $x$ & $x$ & $x$ & $x$ & \\
\hline Review treatment/management & & $x$ & $x$ & $x$ & $x$ & $x$ & $x$ & $x$ & $x$ & $x$ & $x$ & $x$ & $x$ & $x$ \\
\hline ECG for safety & & $x$ & & $x$ & & $x$ & & & $x$ & & & $x$ & $x$ & $x$ \\
\hline Adverse event evaluation & & $x$ & $x$ & $x$ & $x$ & $x$ & $x$ & $x$ & $x$ & $x$ & $x$ & $x$ & $x$ & $x$ \\
\hline \multicolumn{15}{|l|}{ 4. Laboratory tests for safety } \\
\hline Full blood count, liver function tests, creatinine ${ }^{4}$ & & $x$ & & & & & & & $x$ & & & & $x$ & \\
\hline Pregnancy test for women of reproductive age & $x$ & & & & & & & & & & & & & \\
\hline \multicolumn{15}{|l|}{ 5. SARS-CoV-2 testing } \\
\hline PCR for SARS-CoV-2 & & $x$ & & $x$ & & $x$ & & & $x$ & & & $x$ & $x$ & $x$ \\
\hline \multicolumn{15}{|l|}{ 6. Sample for future testing } \\
\hline Oropharyngeal swabs ${ }^{5}$ & & $x$ & & & & & & & $x$ & & & & $x$ & \\
\hline Blood/serum ${ }^{5}$ & & $x$ & & & & & & & $x$ & & & & $x$ & \\
\hline
\end{tabular}

${ }^{1}$ Time frame for test results: Results of tests done within $72 \mathrm{~h}$ of enrolment will be used

${ }^{2}$ Treatment duration: If the patient is still on admission after day 14 , standard treatment will continue in all arms of the study while study medications stop on day 7 for chloroquine and hydroxychloroquine and on day 14 for lopinavir/ritonavir ${ }^{3}$ Clinical data to be collected: See the "Efficacy assessments" section for details

${ }^{4}$ Laboratory tests for safety: Blood-haemoglobin, white cell count, platelets, glucose, creatinine, bilirubin, alanine aminotransferase, aspartate aminotransferase

${ }^{5}$ Samples for future research: To be used when additional SARS-CoV-2 tests become available

or an inappropriate measuring device (i.e. inappropriatesized BP cuff).

\section{Participant timeline $\{13\}$}

The participant timeline is shown in Fig. 1.

\section{Sample size $\{14\}$}

The sample size of 200 per arm has been calculated assuming an odds ratio $(O R)$ of $2,85 \%$ power and a 2 tailed test at level $\alpha=0.05$ and allowing for approximately $10 \%$ of participants to be lost to follow-up. The

Table 5 NEW score

\begin{tabular}{|c|c|c|c|c|c|c|c|}
\hline \multirow[t]{2}{*}{ Physiologic parameters } & \multicolumn{7}{|l|}{ Score } \\
\hline & 3 & 2 & 1 & 0 & 1 & 2 & 3 \\
\hline Respiratory rate & $\leq 8$ & & $9-11$ & $12-20$ & & $21-24$ & $\geq 25$ \\
\hline Oxygen saturation & $\leq 91$ & $92-93$ & $94-95$ & $\geq 96$ & & & \\
\hline Supplemental oxygen & & Yes & & No & & & \\
\hline Temperature & $\leq 35.0$ & & $35.1-36.0$ & $36.1-38.0$ & $38.1-39.0$ & $\geq 39.1$ & \\
\hline Systolic BP & $\leq 90$ & $91-100$ & $101-110$ & $111-219$ & & & $\geq 220$ \\
\hline Heart rate & $\leq 40$ & & $41-50$ & $51-90$ & $91-110$ & $111-130$ & $\geq 131$ \\
\hline Level of consciousness* & & & & A & & & VPU \\
\hline
\end{tabular}

*Level of consciousness: alert (A), arousable only to voice $(\mathrm{V})$ or pain $(\mathrm{P})$, and unresponsive $(\mathrm{U})$ 
Table 6 Normal reference ranges for vital signs and safety assessments

\begin{tabular}{ll}
\hline Parameter & Reference range \\
\hline Temperature & $36.1-37.2^{\circ} \mathrm{C}$ \\
Pulse rate & $60-100$ beats/min \\
Respiratory rate & $12-16 \mathrm{cycles} / \mathrm{min}$ \\
Systolic blood pressure & $90-130 \mathrm{mmHg}$ \\
Diastolic blood pressure & $70-90 \mathrm{mmHg}$ \\
Haemoglobin & $12-17.5 \mathrm{~g} / \mathrm{dl}$ \\
White blood cell count & $4.00-11.0 \times 109 / \mathrm{l}$ \\
Platelet count & $150,000-450,000 / \mu l$ \\
Creatinine & $0.5-1.1 \mathrm{mg} / \mathrm{dl}$ \\
Random glucose & $76-150 \mathrm{mg} / \mathrm{dl}$ \\
Total bilirubin & $0-1.0 \mathrm{mg} / \mathrm{dl}$ \\
ALT & $0-40 \mathrm{u} / \mathrm{l}$ \\
AST & $0-37 \mathrm{i} . \mathrm{u} / \mathrm{l}$ \\
\hline
\end{tabular}

odds ratio represents the odds of improvement in the ordinal scale for treatment relative to control.

\section{Recruitment $\{15\}$}

It is anticipated that patients with COVID-19 will present to the participating hospitals which are designated COVID-19 treatment centres. Therefore, no other efforts to recruit potential subjects are needed.

\section{Assignment of interventions: allocation}

\section{Sequence generation $\{16 a\}$}

Prior to study initiation, a randomization list will be generated (using the statistical programming language $R$, version 3.6.3 to enable random assignment to each of the trial arms at each of the study sites using an equal allocation ratio (for instance 1:1:1:1 for four arms)). If arms are added to or dropped from the trial, randomization will proceed with an equal probability of assignment to each of the remaining arms.

Randomization will be stratified by site and at each site by severity of illness at enrollment yielding two strata: (1) asymptomatic (no clinical symptoms or signs) and (2) symptomatic (presence of clinical symptoms and/ or signs). The $\mathrm{R}$ code used to generate the randomization lists will store the seed so that the generated lists are reproducible.

The randomization list will be generated using permuted block randomization with two block sizes, with the larger block size chosen so that it is not a multiple of the smaller block. Using permuted block randomization rather than simple randomization will avoid the possibility of long periods of time during which participants are not allocated to one or more arms of the study-a scenario which could introduce bias to the study due to time effects. Two block sizes would make it more difficult for clinicians (who will be unaware of the block sizes) to guess any part of the allocation sequence, thus, reducing the potential for the introduction of selection bias by well-meaning staff members at study sites.

\section{Concealment mechanism \{16b\}}

Eligible patients will be allocated to receive medication in individually numbered packs, according to the sequential order of the randomization list. These will be packaged by an unblinded team at the Lagos State central pharmacy lead by the clinical research pharmacist. These personnel are otherwise not involved in the management/care and assessment of the study participants. Neither the names of the investigational drugs nor the arm of the study will be included in the medication packs.

After assignment to interventions, trial participants, care providers and outcome assessors will remain blinded through the use of placebo that will have the same physical formulation, route of administration and dosing schedule.

\section{Implementation $\{16 c\}$}

The allocation sequence will be generated by a member of the trial team who will be based away from the trial sites and will not be involved in care of the participants, outcome assessment or management/analysis of unblinded data.

Clinical research assistants will enrol participants and assign intervention medication packs in sequential order of the randomization list and stratification.

\section{Assignment of interventions: blinding}

Who will be blinded $\{17 \mathrm{a}\}$

Trial participants, the clinical care team, research team, outcome assessors, data management personnel and data analysts will be blinded.

\section{Procedure for unblinding if needed $\{17 \mathrm{~b}\}$}

Individual envelopes containing the arm of the study and trial drugs enclosed in each numbered medication pack will be prepared for emergency unmasking in cases of serious adverse events (SAEs) and serious and unexpected suspected adverse reactions (SUSARs).

To unblind a participant, the study physician at the concerned study site will send the completed unblinding request form via email or manually to the clinical oversight lead at the study site. The study site pharmacist will be notified in the communication as well. The clinical oversight lead will notify the principal investigator and authorize unblinding.

Once the authorization request is approved, the site pharmacist will proceed to release the coded envelope for the research participant concerned. The site 


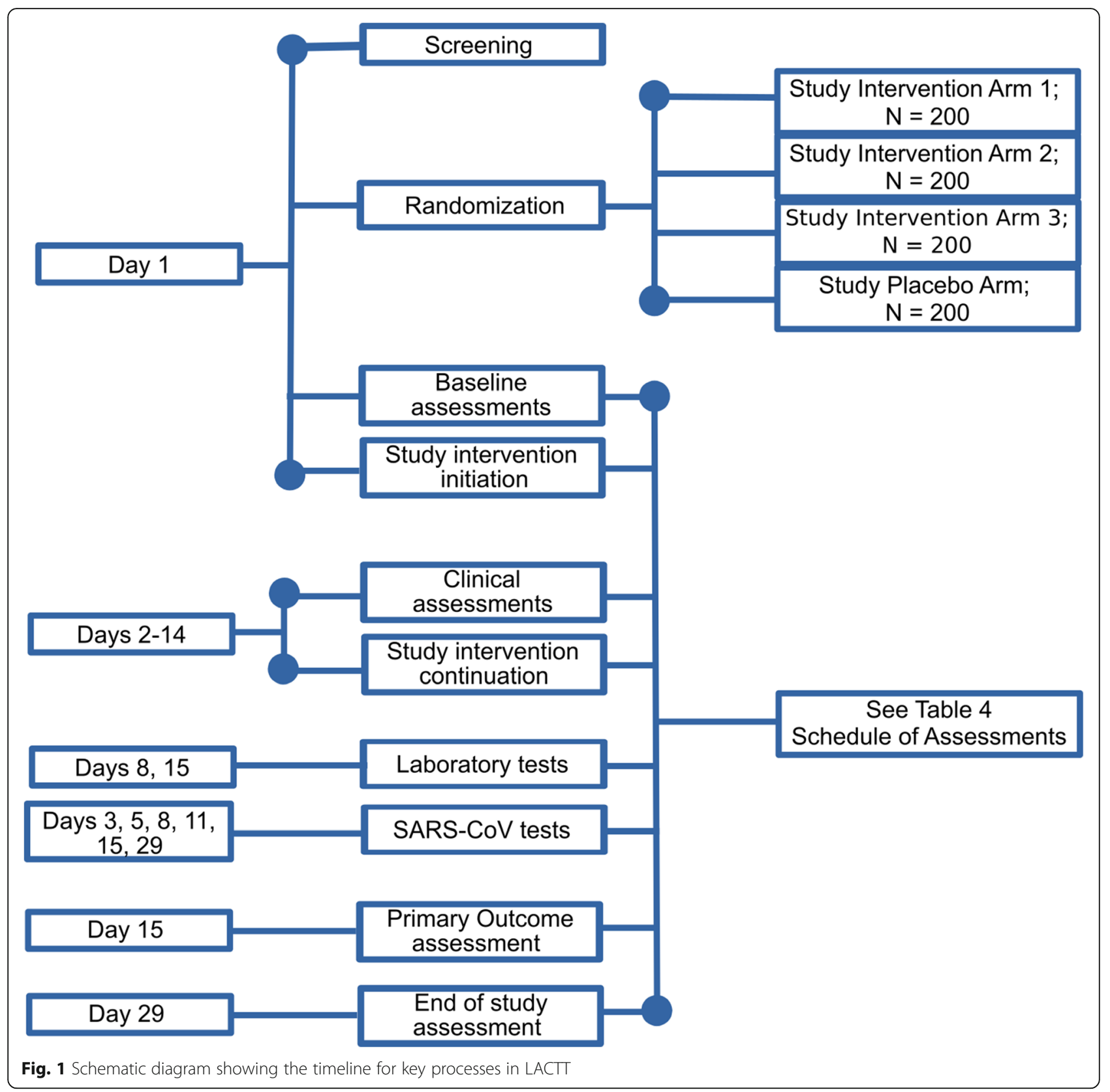

pharmacist will also file a copy of the unblinding request form in the pharmacy records for the study. The site pharmacist will ensure that the treatment assignment of the research participant concerned is shared with the study physician for the study site alone who would in turn take the necessary actions for the management of the patient and reporting adverse events and SUSARs.

\section{Data collection and management}

Plans for assessment and collection of outcomes $\{18 a\}$

Participant data will be collected using case reporting forms (on mobile tablets with REDCap ${ }^{\circ}$ ) to be completed by trained clinical research assistants at baseline all through day 15 and then day 29. The use of REDCap ${ }^{\circ}$ with periodic data reviews by a data management and auditing team will help the research team maintain data quality.

The schedule of assessments is shown in Table 4 and illustrated in Fig. 1. See Supplements for case reporting forms.

\section{SOC available in each participating study site}

Data on the standard of care available and provided to each participant will be summarized for each study site and the entire study population. 
Plans to promote participant retention and complete follow-up $\{18 b\}$

Research participants in the treatment and control arms will be encouraged by the research team and given access to COVID-19 educational resources during the study. In addition, participants will receive communication from the research welfare team to attend to any concerns or questions participants may have during the study.

For participants who deviate or discontinue from the protocol, all outcome data relevant to the study prior to deviation or discontinuation from the study protocol will be collected.

\section{Data management $\{19\}$}

The site investigator is responsible for assuring that the data collected is complete, accurate and recorded in a timely manner. Study data will be entered into REDCap ${ }^{\circ}$ using tablets. Source documentation (the point of initial recording of information) will be signed and dated by the person recording and/or reviewing the data and will support the data transferred to REDCap ${ }^{\circ}$. The data entered into REDCap ${ }^{\circledast}$ will be compared with source documentation on a regular periodic bases as part of data quality assurance.

Due to the biohazard of SARS-COV-2 virus contamination, any original written source documents created at the bedside (i.e. in the 'red zone') will be treated as potentially infectious unless an effective means of decontamination can be instituted. Where possible, photographs, digital scans or other electronic data capture methods may be obtained instead of transporting the original written source documents from the 'red zone'. Interview of subjects will be used for obtaining medical history.

\section{Confidentiality $\{27\}$}

All records will be kept confidential to the extent provided by Lagos State University Teaching Hospital Health Research Ethics Committee (LASUTH HREC) regulations. The study monitors and other authorized representatives of the Sponsor may inspect all documents and records required to be maintained by the investigator, including but not limited to, medical records. Records will be kept locked and all computer entry will be done with coded numbers only. Clinical information will not be released without written permission of the subject, except as necessary for monitoring by LASUTH HREC.

Plans for collection, laboratory evaluation and storage of biological specimens for genetic or molecular analysis in this trial/future use $\{33\}$

Any use of the sample or data for secondary research purposes, however, will be presented in a separate protocol and require separate HREC approval. Each sample will be labelled only with participant ID to protect subject confidentiality.

Each participant can withdraw consent at any time by notifying the study physicians or nurses in writing. If the subject subsequently changes his/her decision, the samples will be destroyed if the samples have not been used for research or released for a specific research project.

\section{Statistical methods}

Statistical methods for primary and secondary outcomes \{20a\}

The statistical methods are based mainly on the WHO Master Protocol [42], as outlined below.

\section{Statistical methods for analyzing the primary outcome}

The primary hypothesis is that the clinical status measured by a 7-point ordinal scale for each of the investigational arms $(1,2,3)$ will be different from that of the placebo arm.

Boschloo's test will be used to evaluate this primary hypothesis in all randomized participants (intention-totreat analysis). For each comparison of a given arm to control, only participants concurrently randomized to those two arms will be included. Statistical significance will be claimed if the two-sided $P$-value is less than the monitoring boundary allocating a total type I error rate of 0.05 across interim analyses. The Boschloo's test is being used instead of Fisher's exact test because the interim results, as well as results within subgroups, will have small sample sizes based on the multiple primary outcomes within the subgroups.

The ordinal scale will be used to estimate a proportional odds model. The primary hypothesis test will be based on a test of whether the common odds ratio for treatment is equal to one. Nonetheless, estimation and confidence intervals do require the model to be correct. Accordingly, we will evaluate model fit using a goodness-of-fit likelihood ratio test. A stratified hypothesis test to account for baseline severity of disease will be used.

The distribution of severity results will be summarized by treatment arm as percentages, and participants without final outcome data will be excluded from the analysis.

\section{Statistical methods for analyzing secondary outcomes}

The following analyses will be performed for the comparison of each experimental arm to the placebo arm when full enrolment has been achieved. For these analyses, the alpha level used is that of the analysis for the primary outcome when full enrolment has been achieved. 
1. The Mantel-Haenszel odds ratio estimator and test statistic will be applied to the strata defined by clinical signs and symptoms (asymptomatic versus symptomatic).

2. Differences in time-to-event endpoints (e.g. time to negative SARS-CoV-2 status) by treatment will be summarized with Kaplan-Meier curves and 95\% confidence bounds.

3. Change in ordinal scale at specific time points will be summarized by proportions (e.g. proportion who have a 1-, 2-, 3- or 4-point improvement or worsening).

4. Duration of event (e.g. duration of mechanical ventilation) will be summarized according to median days with quartiles.

5. Incidence data (e.g. incidence of new oxygen use) will be summarized as a percent with $95 \%$ confidence intervals.

6. Categorical data (e.g. 28-day mortality or ordinal scale by day) will be summarized according to proportions with confidence intervals on the difference or odds ratios for a binary or multiple category scale, respectively.

7. Logistic regression analyses will be undertaken to assess the effect of covariates on outcomes.

\section{Interim analyses $\{21 \mathrm{~b}\}$ \\ Interim analyses}

Interim safety analyses by the Data Coordinating Centre and the DSMB will occur at approximately $25 \%, 50 \%$ and $75 \%$ of total enrolment. Safety analyses will evaluate serious AEs by treatment arm and test for differences using a Pocock spending function approach with a onesided type I error rate of 0.025 . This approach is less conservative than what will be used to test for early efficacy results because proving definitive harm of the experimental agents is not the focus of this study. Pocock stopping boundaries at the looks described correspond to $z$-scores of 2.37, 2.37 and 2.36. This contrasts with the $z$-score stopping boundaries for the Lan-DeMets spending function that mimics O'Brien-Fleming boundaries: $4.33,2.96$ and 2.36 . The unblinded statistical team will prepare these reports for review by the DSMB.

\section{Interim efficacy review}

The Lan-DeMets spending function analogue of the O'Brien-Fleming boundaries will be used to monitor the primary endpoint as a guide for the DSMB for an overall two-sided type I error rate of 0.05 . Interim efficacy analyses will be conducted after the DSMB has selected the primary efficacy endpoint at approximately 25\%, 50\% and $75 \%$ of total information.

\section{Conditional power}

Conditional power will be presented as an additional guide to the DSMB. Conditional power allows computation of the probability of obtaining a statistically significant result by the end of the trial given the data accumulated thus far, incorporating and assuming a hypothesized treatment effect (e.g. the treatment effect assumed for sample size determination) thereafter. If conditional power is less than $20 \%$ under the original trial assumptions, consideration will be given to stopping the trial.

Access to interim results and decision to terminate the trial The unblinded statistical team will prepare the interim result reports for DSMB review and recommendations. Analyses will be presented with blinded codes for treatment arms to protect against the possibility that the DSMB report may fall into the wrong hands. A DSMB charter will further describe procedures and membership. An additional document on statistical issues related to monitoring will be provided to the DSMB prior to interim analyses. The final decision to terminate the trial or an arm of the trial based on interim findings will be made by the DSMB, chaired by an epidemiologist.

\section{Methods for additional analyses (e.g. subgroup analyses) \{20b\} \\ Evaluating safety and tolerability relative to the control arm}

Safety will be evaluated by comparing the proportion of patients with at least one serious adverse event (SAE) for each study arm relative to the control arm, using a twosided Fisher's exact test at alpha $=0.05$. Proportions of specific SAEs will be reported for each study arm. Clopper-Pearson confidence intervals for within-arm proportions will be presented.

We will tabulate the number (\%) of patients with at least one SAE for each study arm. $P$-values comparing each arm to control using two-sided Fisher's exact test at alpha $=0.05$. The number (\%) of patients with specific SAEs by System Organ Class (SOC) and Preferred Term (PT) will also be tabulated. $P$-values comparing each arm to control using two-sided Fisher's exact tests at alpha $=0.05$.

List of SAEs: randomized arm, days from randomization to first $\mathrm{SAE}$ experienced by a subject, subject ID, site, age, days from the start of study drug to $\mathrm{SAE}, \mathrm{SAE}$, severity grade, relatedness to study intervention, outcome (sort by randomized arm, subject ID, SOC and PT and, if a subject has multiple SAEs, days from randomization to SAE). 
Comparing mortality rates of investigational arms to the control arm up to 29 days after randomization

Boschloo's test will be used to evaluate 15-day and 29day clinical status and SARS-CoV-2 viral status in the manner proscribed for the primary and secondary analysis.

Table: Sample size, number (\%) with 15-day 7-point ordinal scale clinical outcome status, number (\%) missing 15-day 7-point ordinal scale clinical outcome status and 15-day SARS-CoV-2 viral status. P-values from Boschloo's test comparing each arm to control.

Table: Sample size, number (\%) with 29-day 7-point ordinal scale clinical outcome status, number (\%) missing 29-day 7-point ordinal scale clinical outcome status and 29-day SARS-CoV-2 viral status. P-values from Boschloo's test comparing each arm to control.

Comparing time to meeting criteria for successful discharge from the COVID-19 treatment centre between participants receiving investigational therapeutics, relative to the control arm

- Differences in median days to becoming eligible for discharge will be tested using the Wilcoxon ranksum test, imputing deaths prior to day 28 as the worst rank, with earlier deaths having a worse rank than later deaths.

- Discharge decisions may not always be based on objective clinical data. For these reasons, time to meeting criteria for successful discharge will be analysed rather than time to actual discharge.

- We will tabulate the sample size, number (\%) who became eligible for discharge within 29 days, mean, median and standard deviation of days to discharge eligibility among those for whom the endpoint is available. $P$-values for test of medians using Wilcoxon rank-sum test.

- Side-by-side plots and boxplots of time to meeting criteria for successful discharge by arm, separately for symptomatic and asymptomatic cases, will be prepared.

\section{Pharmacokinetic assessments of investigational agents}

In general, pharmacokinetic measurements often involve processing (e.g. centrifugation) and testing of blood specimens with techniques or equipment not routinely available or safely performed in most point-of-care laboratory set-ups. These considerations, coupled with limitations on storage, transport and analytical processing of infectious samples falling under Select Agent regulations, could limit these explorations outside the context of a high containment laboratory such as a domestic BSL-4 laboratory in Lagos State.
When possible, pharmacokinetic assessments will include summaries of mean and 95\% confidence intervals for parameters such as the area under the curve (AUC) of concentration versus time, $\mathrm{Cmax}$ and $\mathrm{Cmin}$, clearance time, volume of distribution, half-life and bioavailability.

\section{Methods in analysis to handle protocol non-adherence} and any statistical methods to handle missing data $\{20 \mathrm{c}\}$ The analysis population will be based on intention-totreat. No account will be taken for protocol nonadherence.

For missing data, patterns of missing data will be explored in the primary and secondary outcomes and reported. If required, multiple imputation statistical technique will be used assuming the mechanism of missing data is missing at random (MAR).

Plans to give access to the full protocol, participant-level data and statistical code $\{31 \mathrm{c}\}$

This document is the full protocol. Anyone interested in other data or documentation should contact the corresponding author. Access to participant-level deidentified datasets and statistical codes will be made available on reasonable request to study investigators.

\section{Oversight and monitoring}

Composition of the coordinating centre and trial steering committee $\{5 \mathrm{~d}\}$

The trial will be overseen by a Project Management Team (PMT), a Trial Committee (TC). The PMT will have a zoom meeting approximately every 2 weeks during the recruitment period and then monthly after this. The group will support any decision-making that the trial implementation team need further advice on. The TC will have a chairperson, members and trial collaborators.

\section{Composition of the data monitoring committee, its role and reporting structure $\{21 \mathrm{a}\}$}

The Data Monitoring and Safety Board (DSMB) is responsible for safeguarding the interests of study participants, assessing the safety and efficacy of study procedures, and for monitoring the overall conduct of the study.

The DSMB is required to provide recommendations about starting, continuing and stopping the study. The DSMB is independent of the study sponsor, and members have no competing interests. In addition, the DSMB will make recommendations, as appropriate about:

- Efficacy of the study intervention

- Benefit/risk ratio of procedures and participant burden

- Selection, recruitment and retention of participants 
- Adherence to protocol requirements

- Completeness, quality and analysis of measurements

- Study protocol and consent form amendments

- Performance of individual centres and core labs

- Safety of participants, and notification of and referral for abnormal findings

Further details on the reporting structure of the DSMB can be found within the DSMB Charter in the Supplementary file.

\section{Adverse event reporting and harms $\{22\}$}

Information on all adverse events (AEs) will be recorded on the adverse event form. All clearly related signs, symptoms and results of diagnostic procedures performed because of an $\mathrm{AE}$ will be grouped together and recorded as a single diagnosis. If the $\mathrm{AE}$ is a laboratory abnormality that is part of a clinical condition or syndrome, it will be recorded as the syndrome or diagnosis rather than the individual laboratory abnormality. Each $\mathrm{AE}$ will also be described in terms of duration (start and stop date), severity, association with the study product, action(s) taken and outcome.

\section{Severe adverse events (SAEs)}

Any AE that meets a protocol-defined criterion as a SAE will be submitted immediately (within $24 \mathrm{~h}$ of site awareness) on serious adverse event form to the designated Pharmacovigilance Group at the Lagos State Ministry of Health, the LASUTH HREC and NAFDAC. Other supporting documentation of the event may be requested by the designated Pharmacovigilance Group and will be provided as soon as possible. The designated Medical Monitor will review and assess the SAE for regulatory reporting and potential impact on study subject safety and protocol conduct.

At any time after completion of the study, if the PI or appropriate co-investigator becomes aware of an SAE, the PI or appropriate co-investigator will report the event to the designated Pharmacovigilance Group.

\section{Reporting events to participants}

Subjects will be informed of any severe AEs or SAEs that occur as part of their participation in this trial.

\section{Unanticipated problem (UP) reporting}

To satisfy the requirement for prompt reporting, unanticipated problems (UPs) will be reported using the following timeline:

- UPs that are SAEs will be reported to the HREC and NAFDAC within $24 \mathrm{~h}$ of the investigator becoming aware of the event per the above describe SAE reporting process.
- Any other UP will be reported to the HREC within 3 days of the investigator becoming aware of the problem.

\section{Reporting unanticipated problems to participants}

Subjects will be informed of any UPs that occur as part of their participation in this trial.

Collection forms for AEs and SAEs can be seen in the CRF forms under the Supplementary files.

Frequency and plans for auditing trial conduct $\{23\}$

The Trial Committee and Data Monitoring Board will meet every 6 weeks. The meeting of the TC and DSMB will be independent of the investigators and the study sponsor.

Plans for communicating important protocol amendments to relevant parties (e.g. trial participants, ethical committees) $\{25\}$

Funders, sponsors and LASUTH HREC will be notified routinely and appropriate approvals gained and communicated as required by the research protocol and by the trial sponsor.

\section{Dissemination plans $\{31 \mathrm{a}\}$}

Findings from the study will be disseminated to local and international conference presentations, as well as scientific peer-reviewed journals for publication. A report will also be submitted to the COVID-19 Presidential Task Force and the Lagos State Government through the Lagos State Ministry of Health with key findings from the study and policy implications.

\section{Discussion}

This study is a double-blind randomized controlled trial designed to provide rigorous evidence on the efficacy of chloroquine phosphate, hydroxychloroquine sulphate and lopinavir/ritonavir compared to standard of care only in the treatment of COVID-19. Despite the promising results from in vitro studies, chloroquine has not been shown conclusively to be efficacious in the treatment of COVID-19. However, it is now part of the recommendations for treatment in several countries while awaiting results of planned and on-going clinical trials $[23,36]$. These recommendations have been informed by two main sources-an expert consensus on chloroquine phosphate for treatment of COVID-19 from China and a clinical study undertaken in France [26, 37]. These and one other small study reported from China suggest that chloroquine and hydroxychloroquine prevent worsening of pneumonia, facilitate clearance of the virus and shorten the duration of COVID-19 [24-26, 37]. Broadly, this study is asking three different questions, namely (i) does chloroquine phosphate safely improve patient 
outcome when added to the standard treatment for COVID-19 compared to the addition of placebo; (ii) does hydroxychloroquine sulphate safely improve patient outcome when added to the standard treatment for COVID-19 compared to the addition of placebo?; and (iii) does lopinavir/ritonavir safely improve patient outcome when added to the standard treatment for COVID-19 compared to the addition of placebo.

The study is being undertaken in an emergency context due to the on-going COVID-19 pandemic. In Nigeria, the number of cases identified is rising as testing capacity has increased and deaths are now being recorded [7]. Lagos State accounts for the largest proportion of cases. The current study is of strategic importance for the State in potentially curbing the burden of COVID-19 disease.

Should the current study demonstrate that either of the three intervention drugs is more efficacious than standard therapy alone, the State Ministry of Health will incorporate this in a standard evidence-based guideline for local management of COVID-19. It is anticipated that such a guideline is likely to be adopted nationally.

\section{Trial status}

The submitted protocol is LACTT version 2, May 15, 2020. Recruitment began on June 15, 2020. The approximate date of recruitment completion is November 21, 2021. The trial protocol can be accessed on the Pan African Clinical Trial Register with number PACTR202004801273802.

\begin{abstract}
Abbreviations
AE: Adverse event; ALT: Alanine aminotransferase; CBC: Complete blood count; CNS: Central nervous system; COVID-19: Coronavirus disease 2019; CRF: Case report forms; CTU: COVID-19 treatment unit; DSMB: Drug Safety Monitoring Board; GCP: Good Clinical Practice; GP: Glycoprotein; HCW: Health care worker; HREC : Health Research and Ethics Committee; ICH: International Council for Harmonisation; IgG: Immunoglobulin G; IgM: Immunoglobulin M; IV: Intravenous; IRB: Institutional Review Board; kg: Kilogramme;

Mab: Monoclonal antibody; MEURI: Monitored Emergency Use of Unregistered and Investigational Interventions; $\mathrm{MoH}$ : Ministry of Health; mg: Milligramme; ml: Millilitre; NIMR: National Institute for Medical Research; PK: Pharmacokinetic; PMT: Project Management Team; RCT: Randomized controlled trial;

RNA: Ribonucleic acid; RT-PCR: Reverse transcription-polymerase chain reaction; SAE: Serious adverse event; SAP: Statistical Analysis Plan; SARS: Severe acute respiratory syndrome; SARS-CoV-2: Severe acute respiratory syndrome coronavirus disease 2019; SDCC: Statistical and Data Coordinating Centre; SDSP: Study Data Standardization Plan; SNP: Single nucleotide polymorphisms; SOA: Schedule of activities; SOC: Standard of care; SUSAR: Serious and unexpected suspected adverse reaction; TC: Trial Committee; UP: Unanticipated problem; UPnonAE: Unanticipated problem that is not an adverse event; WBC: White blood cell; WHO: World Health Organization
\end{abstract}

\section{Supplementary Information}

The online version contains supplementary material available at https://doi. org/10.1186/s13063-021-05675-x.

Additional file 1. Research Consent form

Additional file 2. Case Reporting Forms.

Additional file 3. DSMB Charter.

\section{Acknowledgements}

Dr. A. Bowale, Dr. O. Adejumo and Dr. Ayodeji Falana provided information about the current standard therapy for COVID-19 patients in Lagos State.

\section{Authors' contributions $\{31 \mathrm{~b}\}$}

All authors contributed to the design of the study and approved the final version. $\mathrm{AO}, \mathrm{KW}, \mathrm{BO}, \mathrm{EZ}, \mathrm{OAE}$ and SEA conceived the study; $\mathrm{AS}, \mathrm{OE}, \mathrm{KW}$ and YK led on ethics, monitoring and regulatory compliance; $\mathrm{OO}$ contributed to the selection of doses of chloroquine and hydroxychloroquine as well as the unblinding protocol; HA, OAE and SEA led on the study design; HA, OAE and SEA prepared the protocol.

\section{Funding $\{4\}$}

The Lagos State Government will provide funding for this trial. The funding body had no role in the design of the study, data collection, analysis and interpretation of data and in writing the manuscript.

\section{Availability of data and materials $\{29\}$}

The findings of this study will be submitted for publication in a peerreviewed scientific journal. The relevant primary and secondary outcomes will be entered into Pan African Clinical Trials Registry (https://pactr.samrc.ac. za/), and de-identified data will be made available upon request to outside investigators upon reasonable request and review of the scientific merits of their proposed research plan.

\section{Declarations}

Ethics approval and consent to participate $\{24\}$

This trial received ethical approval from the Lagos State Health Research Ethics Committee on April 7, 2020, with reference number LREC/06/10/1344. The trial also received regulatory approval from the National Agency for Food and Drug Administration and Control on May 12, 2020, with reference number NAFDAC/DER/SW/CT\&D//2020/NOL.1. Participants will be provided with essential information about the trial and invited to sign an informed consent document.

Consent for publication $\{32\}$

Written informed consent for publication will be obtained from participants.

\section{Competing interests $\{\mathbf{2 8}\}$}

The authors declare that they have no competing interests.

\section{Author details}

${ }^{1}$ Lagos State Ministry of Health, Alausa, Ikeja, Lagos State, Nigeria. ${ }^{2}$ Lagos State Primary Health Care Board, Lagos, Nigeria. ${ }^{3}$ Nigerian Institute of Medical Research, Lagos, Nigeria. ${ }^{4}$ Lagos State University College of Medicine, Lagos, Nigeria. ${ }^{5}$ Lagos State University Teaching Hospital, Lagos, Nigeria. ${ }^{6}$ Lagos State Health Management Agency, Lagos, Nigeria.

Received: 26 November 2020 Accepted: 30 September 2021

Published online: 04 December 2021

\section{References}

1. World Health Organization. Report of the WHO-China Joint Mission on coronavirus disease 2019 (COVID-19). 2020. [Internet]. [cited 2020 Mar 25]. Available from: https://www.who.int/publications-detail/report-of-the-whochina-joint-mission-on-coronavirus-disease-2019-(covid-19).

2. World Health Organization. WHO Director-General's opening remarks at the media briefing on COVID-19. 2020. [Internet]. [cited 2020 Mar 25]. Available from: https://www.who.int/dg/speeches/detail/who-director-general-sopening-remarks-at-the-media-briefing-on-covid-19\%2D\%2D-11-march-2020.

3. Johns Hopkins CSSE. Coronavirus COVID-19 global cases [Internet]. [cited 2020 July $20^{\text {th }}$ ]. Available from: https://coronavirus.jhu.edu/map.html

4. World Health Organization. Coronavirus disease 2019 (COVID-19) situation report -163. 2020. [Internet]. [cited 2020 July 20 ${ }^{\text {th }}$ ]. Available from: https:// www.who.int/docs/default-source/coronaviruse/situation-reports/20200702covid-19-sitrep-164.pdf?sfvrsn=ac074f58_2.

5. Wu JT, Leung K, Leung GM. Nowcasting and forecasting the potential domestic and international spread of the 2019-nCoV outbreak originating in Wuhan, China: a modelling study. Lancet Lond Engl. 2020;395(10225):689-97. 
6. World Health Organization. Modes of transmission of virus causing COVID19: implications for IPC precaution recommendations. Scientific brief. 2020 [Internet]. [cited $2020 \mathrm{Apr}$ 5]. Available from: https://www.who.int/newsroom/commentaries/detail/modes-of-transmission-of-virus-causing-covid-19implications-for-ipc-precaution-recommendations.

7. Nigeria Centre for Disease Control. Coronavirus disease (COVID-19) pandemic [Internet]. An update on COVID-19 outbreak in Nigeria for week 27. [cited 2020 July $20^{\text {th }}$ ]. Available from: https://www.ncdc.gov.ng/diseases/ sitreps/?cat=14\&name=An\%20update\%20of\%20COVID-19\%20outbreak\%2 0in\%20Nigeria.

8. Gorbalenya AE, Baker SC, Baric RS, de Groot RJ, Drosten C, Gulyaeva AA, et al. The species severe acute respiratory syndrome-related coronavirus: classifying 2019-nCoV and naming it SARS-CoV-2. Nat Microbiol. 2020;5(4): 536-44. https://doi.org/10.1038/s41564-020-0695-Z.

9. Wang M, Cao R, Zhang L, Yang X, Liu J, Xu M, et al. Remdesivir and chloroquine effectively inhibit the recently emerged novel coronavirus (2019-nCoV) in vitro. Cell Res. 2020;30(3):269-71. https://doi.org/10.1038/ s41422-020-0282-0.

10. Loutfy MR, Blatt LM, Siminovitch KA, Ward S, Wolff B, Lho H, et al. Interferon alfacon-1 plus corticosteroids in severe acute respiratory syndrome: a preliminary study. JAMA. 2003;290(24):3222-8. https://doi.org/10.1001/jama.2 90.24.3222.

11. Chu CM, Cheng VCC, Hung IFN, Wong MML, Chan KH, Chan KS, et al. Role of lopinavir/ritonavir in the treatment of SARS: initial virological and clinical findings. Thorax. 2004;59(3):252-6. https://doi.org/10.1136/thorax.2003.012 658.

12. Zhang B, Liu S, Tan T, Huang W, Dong Y, Chen L, et al. Treatment with convalescent plasma for critically ill patients with SARS-CoV-2 infection. Chest. 2020;158(1):e9-e13. https://doi.org/10.1016/j.chest.2020.03.039.

13. Chan KS, Lai ST, Chu CM, Tsui E, Tam CY, Wong MML, et al. Treatment of severe acute respiratory syndrome with lopinavir/ritonavir: a multicentre retrospective matched cohort study. Hong Kong Med J Xianggang Yi Xue Za Zhi. 2003;9(6):399-406.

14. Barnard DL, Hubbard VD, Burton J, Smee DF, Morrey JD, Otto MJ, et al. Inhibition of severe acute respiratory syndrome-associated coronavirus (SARSCOV) by calpain inhibitors and beta-D-N4-hydroxycytidine. Antivir Chem Chemother. 2004;15(1):15-22. https://doi.org/10.1177/095632020401 500102.

15. Tan ELC, Ooi EE, Lin C-Y, Tan HC, Ling AE, Lim B, et al. Inhibition of SARS coronavirus infection in vitro with clinically approved antiviral drugs. Emerg Infect Dis. 2004;10(4):581-6. https://doi.org/10.3201/eid1004.030458.

16. Yamamoto N, Yang R, Yoshinaka Y, Amari S, Nakano T, Cinatl J, et al. HIV protease inhibitor nelfinavir inhibits replication of SARS-associated coronavirus. Biochem Biophys Res Commun. 2004;318(3):719-25. https://doi. org/10.1016/j.bbrc.2004.04.083.

17. Groneberg DA, Poutanen SM, Low DE, Lode H, Welte T, Zabel P. Treatment and vaccines for severe acute respiratory syndrome. Lancet Infect Dis. 2005; 5(3):147-55. https://doi.org/10.1016/S1473-3099(05)70022-0.

18. Mair-Jenkins J, Saavedra-Campos M, Baillie JK, Cleary P, Khaw F-M, Lim WS, et al. The effectiveness of convalescent plasma and hyperimmune immunoglobulin for the treatment of severe acute respiratory infections of viral etiology: a systematic review and exploratory meta-analysis. J Infect Dis. 2015;211(1):80-90. https://doi.org/10.1093/infdis/jiu396.

19. Public Health England. Treatment of MERS-CoV: information for clinicians. Clinical decision-making support for treatment of MERS-CoV patients [Internet]. 2017. [cited 2020 Apr 5]. Available from: https://assets.publishing service.gov.uk/government/uploads/system/uploads/attachment_data/file/ 638628/MERS_CoV_guidance_for_clinicians.pdf.

20. Chan JFW, Chan K-H, Kao RYT, To KKW, Zheng B-J, Li CPY, et al. Broad-spectrum antivirals for the emerging Middle East respiratory syndrome coronavirus. J Infect. 2013;67(6):606-16. https://doi.org/10.1016/j.jinf.2013.09.029.

21. Cheng K-W, Cheng S-C, Chen W-Y, Lin M-H, Chuang S-J, Cheng $\mathrm{H}-\mathrm{H}$, et al. Thiopurine analogs and mycophenolic acid synergistically inhibit the papain-like protease of Middle East respiratory syndrome coronavirus. Antivir Res. 2015;115:9-16. https://doi.org/10.1016/j.antiviral.2014.12.011.

22. Wang $Y$, Sun $Y$, Wu A, Xu S, Pan R, Zeng C, et al. Coronavirus nsp10/nsp16 methyltransferase can be targeted by nsp10-derived peptide in vitro and in vivo to reduce replication and pathogenesis. J Virol. 2015;89(16):8416-27. https://doi.org/10.1128/JVI.00948-15.

23. Interim clinical guidance for adults with suspected or confirmed Covid- 19 in Belgium. 2020. Version 6 [Internet]. [cited 2020 Apr 2]. Available from:
https://epidemio.wiv-isp.be/ID/Documents/Covid19/COVID-19_ InterimGuidelines_Treatment_ENG.pdf.

24. Gao J, Tian Z, Yang X. Breakthrough: chloroquine phosphate has shown apparent efficacy in treatment of COVID-19 associated pneumonia in clinical studies. Biosci Trends. 2020;14(1):72-3. https://doi.org/10.5582/bst.2020.01047.

25. Chen J, Liu D, Liu L, Liu P, Xu Q, Xia L, et al. A pilot study of hydroxychloroquine in treatment of patients with common coronavirus disease-19 (COVID-19). J Zhejiang Univ. 2020; [cited 2020 Mar 25]. Available from: http://subject.med.wanfangdata.com.cn/UpLoad/Files/202003/43f862 5d4dc74e42bbcf24795de1c77c.pdf.

26. Gautret P, Lagier J, Parola P, et al. Clinical and microbiological effect of a combination of hydroxychloroquine and azithromycin in 80 COVID-19 patients with at least a six-day follow up: an observational study [Internet]. [cited 2020 Apr 2]. Available from: https://www.mediterranee-infection.com/ wp-content/uploads/2020/03/COVID-IHU-2-1.pdf.

27. Soto Alonso. Nigeria has chloroquine poisonings after Trump praised drug. Bloomberg.com [Internet]. 2020. [cited 2020 Mar 25]. Available from: https:// www.bloomberg.com/news/articles/2020-03-21/nigeria-reports-chloroquinepoisonings-after-trump-praised-drug.

28. Nigeria Centre for Disease Control. NCDC on Twitter: "\#FactsNotFear @WHO has NOT approved the use of chloroquine for \#COVID19 management. Scientists are working hard to confirm the safety of several drugs for this disease. Please DO NOT engage in self-medication. This will cause harm and can lead to death. \#COVID19Nigeria https://t.co/K6kljq0VtW" / Twitter [Internet]. Twitter. [cited 2020 Mar 25]. Available from: https://twitter.com/ ncdcgov/status/1241006420419641345.

29. Savarino A, Boelaert JR, Cassone A, Majori G, Cauda R. Effects of chloroquine on viral infections: an old drug against today's diseases? Lancet Infect Dis. 2003;3(11):722-7. https://doi.org/10.1016/S1473-3099(03)00806-5.

30. Naarding MA, Baan E, Pollakis G, Paxton WA. Effect of chloroquine on reducing HIV-1 replication in vitro and the DC-SIGN mediated transfer of virus to CD4+ T-lymphocytes. Retrovirology. 2007;4(1):6. https://doi.org/10.11 86/1742-4690-4-6.

31. Keyaerts E, Vijgen L, Maes P, Neyts J, Van Ranst M. In vitro inhibition of severe acute respiratory syndrome coronavirus by chloroquine. Biochem Biophys Res Commun. 2004;323(1):264-8. https://doi.org/10.1016/j.bbrc.2004.08.085.

32. Yan Y, Zou Z, Sun Y, Li X, Xu K-F, Wei Y, et al. Anti-malaria drug chloroquine is highly effective in treating avian influenza A H5N1 virus infection in an animal model. Cell Res. 2013;23(2):300-2. https://doi. org/10.1038/cr.2012.165.

33. Hu TY, Frieman M, Wolfram J. Insights from nanomedicine into chloroquine efficacy against COVID-19. Nat Nanotechnol. 2020;(4):1-3. https://doi.org/1 0.1038/s41565-020-0674-9.

34. Vincent MJ, Bergeron E, Benjannet S, Erickson BR, Rollin PE, Ksiazek TG, et al. Chloroquine is a potent inhibitor of SARS coronavirus infection and spread. Virol J. 2005;2(1):69. https://doi.org/10.1186/1743-422X-2-69.

35. Mackenzie $\mathrm{AH}$. Dose refinements in long-term therapy of rheumatoid arthritis with antimalarials. Am J Med. 1983;75(1A):40-5. https://doi.org/10.1 016/0002-9343(83)91269-X.

36. Nicastri E, Petrosillo N, Bartoli TA, Lepore L, Mondi A, Palmieri F, et al. National Institute for the Infectious Diseases "L. Spallanzani", IRCCS. Recommendations for COVID-19 clinical management. Infect Dis Rep. 2020; 12(1) [cited 2020 Apr 2]. Available from: https://www.ncbi.nlm.nih.gov/ pmc/articles/PMC7097833/.

37. Multicenter collaboration group of Department of Science and Technology of Guangdong Province and Health Commission of Guangdong Province for chloroquine in the treatment of novel coronavirus pneumonia. [Expert consensus on chloroquine phosphate for the treatment of novel coronavirus pneumonia]. Zhonghua Jie He He Hu Xi Za Zhi Zhonghua Jiehe He Huxi Zazhi Chin J Tuberc Respir Dis. 2020;43(3):185-8.

38. Cao B, Wang Y, Wen D, Liu W, Wang J, Fan G, et al. A trial of lopinavirritonavir in adults hospitalized with severe Covid-19. N Engl J Med. 2020; 382(19):1787-99. https://doi.org/10.1056/NEJMoa2001282. https://doi.org/1 $0.1056 /$ NEJMoa2001282.

39. Chen F, Chan KH, Jiang Y, Kao RY, Lu HT, Fan KW, et al. In vitro susceptibility of 10 clinical isolates of SARS coronavirus to selected antiviral compounds. J Clin Virol. 2004;31(1):69-75. https://doi.org/10.101 6/j.jcv.2004.03.003.

40. Wu C-Y, Jan J-T, Ma S-H, Kuo CJ, Juan HF, Cheng YSE, et al. Small molecules targeting severe acute respiratory syndrome human coronavirus. Proc Natl Acad Sci U S A. 2004;101(27):10012-7. https://doi.org/10.1073/pnas.0403596101. 
41. WHO Director-General's opening remarks at the media briefing on COVID19. 2020. [Internet]. [cited 2020 Mar 25]. Available from: https://www.who. int/dg/speeches/detail/who-director-general-s-opening-remarks-at-themedia-briefing-on-covid-19\%2D\%2D-18-march-2020.

42. World Health Organization. Master Protocol. A multi-centre, adaptive, randomized, double-blind, placebo-controlled clinical trial of the safety and efficacy of investigational therapeutics for the treatment of COVID-19 in hospitalized patients. Version Number: 2.0. 2020.

43. World Health Organization. WHO R\&D Blueprint. Novel coronavirus COVID19 therapeutic trial. Synopsis. 2020; Geneva, Switzerland.

\section{Publisher's Note}

Springer Nature remains neutral with regard to jurisdictional claims in published maps and institutional affiliations.

Ready to submit your research? Choose BMC and benefit from:

- fast, convenient online submission

- thorough peer review by experienced researchers in your field

- rapid publication on acceptance

- support for research data, including large and complex data types

- gold Open Access which fosters wider collaboration and increased citations

- maximum visibility for your research: over $100 \mathrm{M}$ website views per year

At $\mathrm{BMC}$, research is always in progress.

Learn more biomedcentral.com/submissions 